\title{
COVID-19 in Pregnancy: Pregnant Women Might be at Greater Risk for Severe COVID-19 Dangal G
}

Pregnancy is a partially immunosuppressed state and this state along with hormonal changes can predispose to infection. Thus, any viral or bacterial respiratory infection like pneumonia can be life-threatening during pregnancy. ${ }^{1}$ Pregnancy related alteration in immunologic response and physiologic changes mainly due to hormones could increase pregnant women's risk for more severe illness from respiratory infections in general. $^{2,3}$

Up till now limited information is available about confirmed SARS-CoV-2 infection in pregnant women worldwide. Among women with SARS-CoV-2 infection, pregnancy was associated with more hospitalization and increased risk for intensive care unit admission, and mechanical ventilation compared with nonpregnant women. Presence of underlying chronic conditions like diabetes and hypertension led to a more pronounced morbidity in them. However, pregnant COVID-19 patients do not appear to have a greater risk for mortality. ${ }^{4}$ However, collection of more consolidated and complete data for pregnant women with SARS-CoV-2 infection, and information about pregnancy outcomes, is needed to fully understand the effects of SARS-CoV-2 infection on maternal and neonatal outcomes and the risk for severe illness in pregnant women. Thus, prevention is to be emphasized and pregnant women should stay healthy and take steps to reduce their risk for getting COVID-19.

During COVID-19 pandemic, stress levels of couples mainly due to social, personal and financial situations; have substantially increased leading to subfertility also. We do not have much data on miscarriages also but it seems to be happening more frequently as a result of various pandemic related factors. Pregnant women should take the same precautions to avoid COVID-19 infection as other people and seek medical care early if there are any similar symptoms. Pregnant women with symptoms of COVID-19 should be prioritized for testing. ${ }^{5}$

Routine antenatal care (ANC) visits need to be modified and a total of three visits with sonographies at 12,18 and 32 weeks is recommended. Use of telemedicine and video consultation instead should be encouraged for ANC checkups. Right now, there is no clear evidence on the ideal mode and timing of delivery of COVID positive women. Cesarean section is reserved for obstetric indications and management has to be individualized by avoiding a difficult and prolonged labor. However, a separate labor room and dedicated theatre is required with all universal precautions for prevention of COVID-19 transmission. Early exclusive breast feeding and bonding helps a baby to thrive. Women with COVID-19 can breastfeed if they wish to do so. However, they should practice respiratory hygiene, wear a mask, wash hands and sanitize the surfaces around the mother. ${ }^{6}$ Access to effective contraception is one of the most cost-effective interventions to reduce maternal mortality by preventing an unplanned pregnancy, thereby protecting an individual's physical and mental health. Contraceptive advice and services should be given as per standard and long-acting reversible contraceptive or Depo-Provera should be considered if the patient wished before discharging her to eliminate in-person postnatal visits. ${ }^{5,6}$ COVID-19 also seems to be a transient risk factor for venous thromboembolism. It has mental health implications and gender-based violence has also been on the rise. ${ }^{7}$

One of the worrisome issues is pregnant and lactating women being excluded from majority of COVID-19 researches and this exclusion is potentially harmful as we do not know the safety profile of the trial drug in them and whether drug transfers into breast milk. Rather than excluding them from research, we must protect them through research. The most worrisome of all is the exclusion of pregnant and lactating women from most clinical trials of potential coronavirus vaccines. This is condemnable as this inappropriate act underscores the need of pregnant and lactating women to receive coronavirus vaccination when it becomes available and this can definitely be seen as a valid ethical concern, dilemma and dispute on the part of vaccine developers and other stakeholders. ${ }^{8}$ 
In summary, COVID-19 is a pandemic affecting every group of people including pregnant and lactating women. There is lower immunity in pregnancy possibly leading to aggravation of symptoms. Protection of pregnant women from COVID-19 by adopting proper preventive strategies is of utmost importance. There is no proven vertical transmission from the limited data available. ${ }^{7}$ Newborn has to be kept in isolation and breastfeeding can be done with routine preventive precautions. All should follow the updated national and international guidelines and be beware of myths like Nepalese immunity is strong, weaker stain of virus is here and benefit of BCG vaccination, etc. Pregnant women should be given equal opportunity to be included in COVID-19 clinical trials including the remdesivir, convalescent plasma and vaccine trials.

Prof. Dr. Ganesh Dangal,

Department of Obstetrics and Gynecology, Kathmandu Model Hospital, Exhibition Road, Kathmandu, Nepal.

Email: ganesh.dangal@gmail.com

\section{REFERENCES}

1. Mathad JS, Gupta A. Pulmonary infections in pregnancy. Semin Respir Crit Care Med. 2017; 38:174-84. https://doi.org/10.1055/s-0037-1602375

2. Ramsey PS, Ramin KD. Pneumonia in pregnancy. Obstet Gynecol Clin North Am. 2001;28:553-69. https://doi.org/10.1016/S0889-8545(05)70217-5

3. Rasmussen SA, Kissin DM, Yeung LF, MacFarlane K, Chu SY, Turcios-Ruiz RM, et al. Pandemic Influenza and Pregnancy Working Group. Preparing for influenza after 2009 H1N1: special considerations for pregnant women and newborns. Am J Obstet Gynecol. 2011;204(Suppl 1):S13-20. https://doi. org/10.1016/j.ajog.2011.01.048

4. Ellington S, Strid P, Tong VT, Woodworth K, Galang RR, Zambrano LD, et al. Characteristics of Women of Reproductive Age with Laboratory-Confirmed SARS-CoV-2 Infection by Pregnancy Status - United States, January 22-June 7, 2020. MMWR Morb Mortal Wkly Rep. 2020;69:769-75. http://dx.doi. org/10.15585/mmwr.mm6925a1

5. World Health Organization. COVID-19: Resources for Pregnancy, Childbirth, Postnatal Care [Internet]. Geneva: Switzerland. [2017 May 17]. Available at: https://www.who.int/maternal_child_adolescent/links/covid-19-mncah-resources-for-pregnancy-childbirth-postnatalcare/en/

6. Nepal Society of Obstetricians and Gynecologists. NESOG Clinical Practice Guidance for Management of Reproductive Health during COVID-19 Pandemic. Kathmandu: NESOG; 2020.

7. RCOG. Coronavirus (COVID-19) infection and pregnancy [Internet]. London: UK. [2020 July 30]. Available at: https://www.rcog.org.uk/coronaviruspregnancy.

8. Smith DD, Pippen JL, Adesomo AA, Rood KM, Landon MB, Costantine MM. Exclusion of Pregnant Women from Clinical Trials during the Coronavirus Disease 2019 Pandemic: A Review of International Registries. Am J Perinatol. 2020 Jun;37(8):792-799. doi: 10.1055/s-0040-1712103 